\title{
Dépistage néonatal de la mucoviscidose également en Suisse - dès le premier janvier 2011
}

Jürg Barben ${ }^{a}$, Toni Torresani ${ }^{b}$, Martin H. Schönic, Sabina Gallati , Matthias Baumgartnere et task force dépistage néonatal de la mucoviscidose* $^{*}$

a Président du Swiss Working Group for Cystic Fibrosis (SWGCF), secrétaire de la société suisse de pneumologie pédiatrique (SSPP), médecin-chef de pneumologie/allergologie, Ostschweizer Kinderspital St Gall

b Directeur du dépistage néonatal suisse et du Proteinhormonlabor Endokrinologie, cliniques pédiatriques universitaires Zurich

c Médecin-chef de la pédiatrie ambulatoire, clinique pédiatrique universitaire, Berne

d Directrice du département de génétique humaine, clinique pédiatrique universitaire, Berne

e Directeur du département des maladies métaboliques, cliniques pédiatriques universitaires Zurich

* Autres membres: Carmen Casaulta (Berne), Anne Mornand (Genève), Ralph Fingerhut (Zurich), Gaudenz Hafen (Lausanne), Alexander Möller (Zurich), Nicolas Regamey (Berne)

Traduction: Rudolf Schlaepfer, La Chaux-de-Fonds

Correspondance: PD Dr Jürg Barben Président SWGCF Leitender Arzt Pneumologie/ Allergologie

Ostschweizer Kinderspital

CH-9006 Saint-Gall

Tél. 0712437111

Fax 0712437390

juerg.barben@kispisg.ch
Nous disposons en Suisse, depuis plus de 40 ans, d'un dépistage néonatal pour de nombreuses maladies métaboliques congénitales, communément appelé «test de Guthrie». Le 4e jour de vie on prélève du talon de chaque nouveau-né en Suisse un petit échantillon de sang qu'on laisse sécher sur un papier-filtre. Depuis l'automne 2005 l'évaluation est centralisée dans le laboratoire de dépistage néonatal de la Clinique pédiatrique universitaire de Zurich. Jusqu'ici six maladies traitables sont dépistée et, dès le $1^{\text {er }}$ janvier 2011, la mucoviscidose fera partie de ce dépistage (tabl. 1).

\section{Tableau 1}

Maladies dépistées actuellement par le Programme national de dépistage systématique néonatal:

Phénylcétonurie

Galactosémie (déficit en transférase, kinase, épimérase)

Déficit en Biotinidase

MCADD (déficit en déshydrogénase des Acyl-CoA

à chaîne moyenne)

Hypothyroïdie congénitale

Hyperplasie congénitale des surrénales

Nouveau: Mucoviscidose

Avec une incidence d'environ 1:2500, la mucoviscidose est le trouble du métabolisme congénital le plus fréquent en Suisse, ce qui signifie que la mucoviscidose est plus fréquente que toutes les maladies testées jusqu'ici. Exception faite de l'ileus méconial, la mucoviscidose connaît elle aussi un intervalle asymptomatique immédiatement après la naissance. Le diagnostic, souvent tardif, après des mois voire des années, se basait jusqu'ici sur des symptômes cliniques (toux récidivante ou bronchite chronique, troubles de la croissance staturo-pondérale, stéatorrhée, douleurs abdominales chroniques, rhinosinusite chronique, polypes nasaux, etc.). Durant ces dernières années, les possibilités thérapeutiques se sont considérablement améliorées et l'espérance de vie a augmenté de façon dramatique. D'après le registre américain de patients avec mucoviscidose, l'âge médian est en moyenne déjà de presque 40 ans et en Grande-Bretagne on estime l'âge de survie moyen à 50 ans pour les patients nés au XXI ${ }^{e}$ siècle [1].

Depuis 1979, avec la trypsine immunoréactive (IRT) nous disposons d'une méthode simple et très fiable pour le dépistage de la mucoviscidose dans le sang, facilement applicable chez le nouveau-né [2]. Les premiers grands programmes de dépistage de la mucoviscidose ont été lancés déjà en 1981 en Nouvelle Zélande et en Australie [3]. A ce jour un programme de dépistage néonatal de la mucoviscidose existe dans tous les états américains et dans un grand nombre de pays européens comme la Grande-Bretagne, l'Irlande, l'Ecosse, la France, l'Autriche et la Pologne ainsi que dans beaucoup de régions d'Italie et d'Espagne $[4,5]$. Entre temps des recommandations américaines et européennes ont été également édictées pour le développement, la mise en pratique et la gestion de tels programmes $[6,7]$.

\section{Quels sont les avantages du dépistage néonatal de la mucoviscidose?}

Jusqu'il y a une dizaine d'années il n'existait pas d'études contrôlées démontrant l'avantage du dépistage précoce de la mucoviscidose. De nombreuses études publiées ces dernières années, montrent par contre que le diagnostic précoce permet une amélioration de l'état nutritionnel et de la croissance, un meilleur développement cérébral (grâce à une substitution adéquate en vitamine E), moins de maladies et de séjours à l'hôpital et, en partie, également une meilleure fonction pulmonaire $[1,8]$. Certaines études ont aussi démontré une survie prolongée. Tous les programmes de dépistage soulignent le grand bénéfice psychologique, le diagnostic précoce abrégeant le temps de souffrance et d'incertitude des parents. Le diagnostic précoce permet en outre un planning familial réfléchi et la possibilité d'un diagnostic prénatal pour la fratrie à venir, en cas de nouveau désir de grossesse [4].

\section{Quels sont les inconvénients?}

Tout examen de dépistage connaît des résultats faux négatifs et faux positifs. Par l'introduction d'un procédé en deux étapes (fig. 1), les faux positifs sont limités au minimum. Le but est de viser un équilibre optimal afin de dépister précocément le plus possible d'enfants avec une mucoviscidose (= bonne sensibilité), en ne retenant que ceux qui développent effectivement une mucoviscidose symptomatique (= bonne spécificité). Le test à la sueur précisera finalement la présence d'une mucoviscidose avec ses symptômes typiques (mucoviscidose classique). Le délai jusqu'à l'obtention de ce résultat n'est pas perçu uni- 
formément par les parents. Le taux de faux négatifs, c'est-à-dire les enfants ayant une mucoviscidose et n'ayant pas été décelés par le test de dépistage sont estimés aux USA à 2-4\% [4]. Cela dépend essentiellement du nombre de mutations génétiques, estimées actuellement à 1600, englobées dans le test de dépistage. Mais cela signifie aussi qu'à l'avenir il faudra quand même penser, en présence de symptômes évocateurs, à une mucoviscidose, car même le plus performant des tests de dépistage ne pourra déceler tous les enfants atteints de cette maladie. Plus grand est le nombre de mutations recherchées, plus grand sera le nombre décelé de formes frustes de mucoviscidose (formes atypiques), qui ne développeront peut-être des symptômes qu'à l'âge de 20 ou 30 ans. Pour cette raison, en Suisse, ne sont identifiées que les sept mutations génétiques les plus fréquentes de la mucoviscidose. Un autre inconvénient potentiel est qu'un enfant diagnostiqué précocément soit infecté, lors d'un séjour dans un centre pour enfants avec mucoviscidose, par des germes avec lesquels il ne serait jamais entré en contact en dehors d'un tel centre. Les recommandations d'hygiène dans les hôpitaux se justifient donc pleinement. Tous les tests de dépistage détectent aussi des porteurs sains d'une mutation pour la mucoviscidose (test de dépistage positif mais test à la sueur normal), condition que certaines personnes ne souhaitent pas connaître.

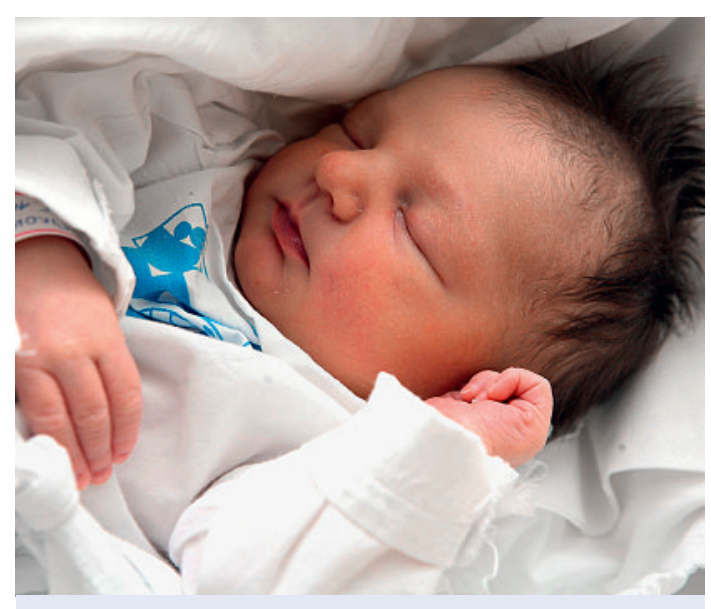

Le diagnostic précoce permet une amélioration de l'état nutritionnel et de la croissance, un meilleur développement cérébral, moins de maladies et de séjours à l'hôpital et, en partie, également une meilleure fonction pulmonaire.

\section{Comment se déroule exactement le test de dépistage pour la mucoviscidose?}

Lorsqu'on constate une valeur élevée de l'IRT, on cherche dans la même goutte de sang, par analyse de l'ADN, les sept mutations génétiques les plus fréquentes en Suisse. Si ce deuxième test est également positif (découverte d'au moins une mutation géné-

Figure 1

Algorithme du dépistage néonatal de la mucoviscidose.

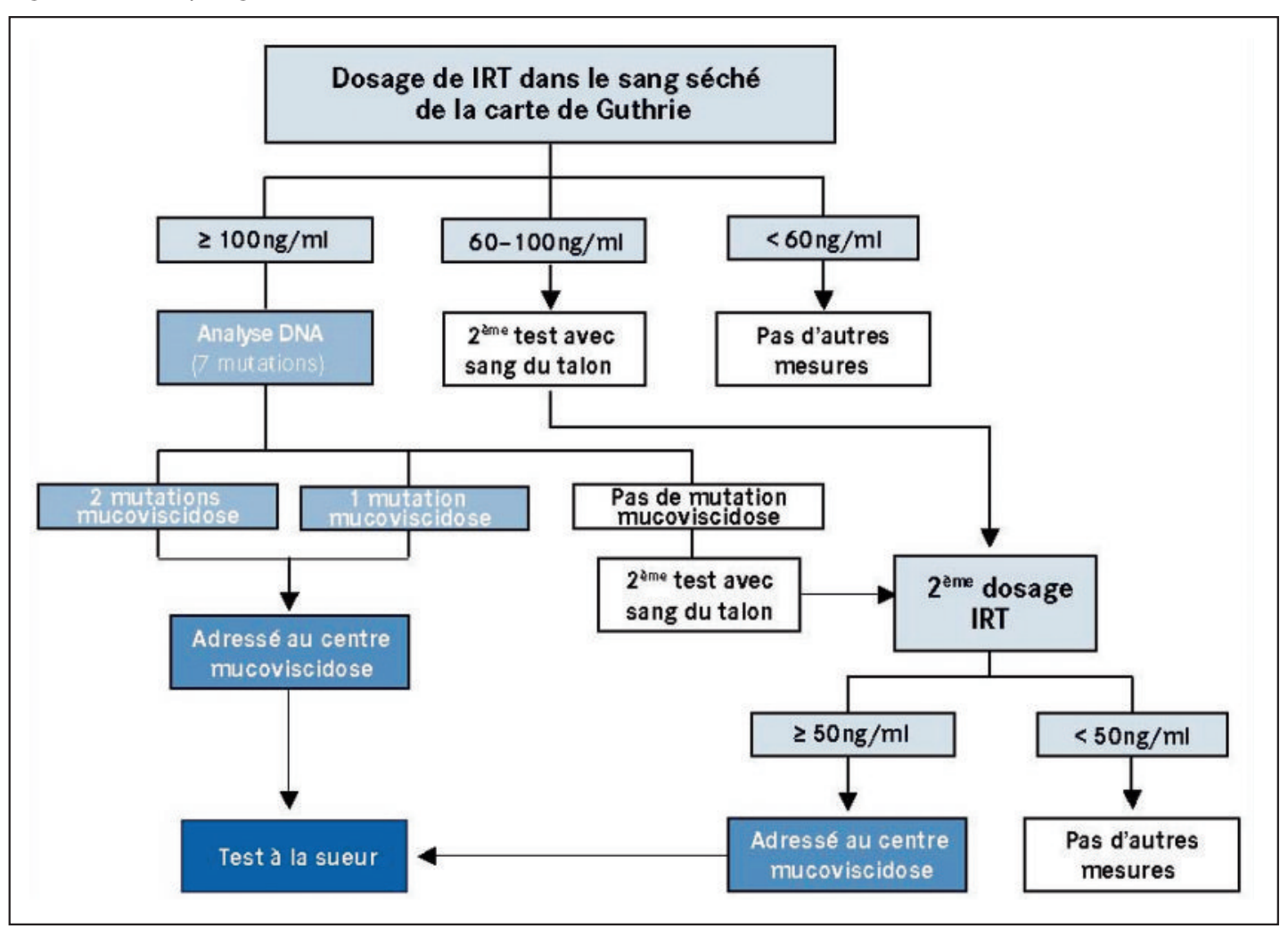


tique), le dépistage est considéré comme positif. Le dépistage néonatal étant une analyse proposée de manière systématique selon la Loi fédérale sur l'analyse génétique humaine (LAGH), le consentement écrit des parents n'est pas nécessaire. Si le test de dépistage fait suspecter une mucoviscidose, un des huit centres pédiatriques d'étude de la mucoviscidose sera informé. Il convoquera les parents et l'enfant pour des investigations complémentaires. Lorsque l'analyse de
En raison de l'importance de cet article aussi bien pour les pédiatres que pour un large public de médecins, les rédactions du BMS et de la revue Paediatrica ont décidé d'un commun accord de publier ce papier dans les deux revues. La référence de cet article dans la revue Paediatrica est Paediatrica 2010;21(5):40-41 (version française).

Nous remercions la rédaction de Paediatrica de nous avoir fourni la traduction française de cet article.

\section{Par l'introduction d'un procédé en deux étapes, les faux positifs sont limités au minimum.}

l'ADN est négative ou le test IRT à la limite de la norme, un deuxième test de Guthrie sera demandé au pédiatre ou à la sage-femme responsable; par analogie à la démarche établie pour le dépistage néonatal. Si, lors de ce deuxième test IRT, la limite de $50 \mathrm{ng} / \mathrm{ml}$ est dépassée, un centre pour enfants avec mucoviscidose sera informé afin de procéder aux examens nécessaires (fig. 1).

Dans un des huit centres pédiatriques de la mucoviscidose, les examens complémentaires pour confirmer ou écarter le diagnostic de mucoviscidose, selon les directives internationales $[9,10]$ (test à la sueur, examens génétiques dans le sang, etc.), ne seront effectués qu'avec le consentement des parents. Un test à la sueur positif confirme le diagnostic. S'il est négatif, aucun autre examen ne sera pratiqué. Ces enfants sont soit sains, soit porteurs sains d'une mutation pour la mucoviscidose. Il peuvent aussi avoir une mucoviscidose atypique, qui ne se manifestera qu'à l'âge adulte et qui aura généralement un décours bénin.

Les parents sont informés du possible état de porteur, respectivement de la présence d'une variante atypique, bénigne de la mucoviscidose et on leur proposera un conseil génétique détaillé à ce sujet par un des centres de génétique officiels en Suisse; ils recevront aussi un aide-mémoire. Avec le consentement des parents, le pédiatre ou le médecin de famille sera informé, ce qui lui permettra de mettre en route les investigations nécessaires dès qu'apparaîtront des symptômes évocateurs.

Le projet pilote actuel est reconnu par l'Office fédéral de la santé publique (OFSP) pour une durée de 2 ans. Si l'expérience s'avère positive, nous demanderons auprès de l'OFSP l'inclusion définitive de la mucoviscidose dans le programme de dépistage néonatal, afin d'en garantir la poursuite.

\section{Références}

1 Balfour-Lynn IM. Newborn screening for cystic fibrosis: evidence for benefit. Arch Dis Child. 2008;93:7-10.

2 Crossley JR, Elliott RB, Smith PA. Dried-blood spot screening for cystic fibrosis in the newborn. Lancet. 1979;1:472-4.

3 Massie J, Clements B, Australian Paediatric Respiratory Group. Diagnosis of cystic fibrosis after newborn screening: the Australasian experience - twenty years and five million babies later: a consensus statement from the Australasian Paediatric Respiratory Group. Pediatr Pulmonol. 2005;39:440-6.

4 Grosse SD, Boyle CA, Botkin JR et al. Newborn screening for cystic fibrosis: Evaluation of benefits and risks and recommendations for state newborn screening programs. MMWR Recomm Rep 2004;53(RR-13):1-36.

5 Southern KW, Munck A, Pollitt R et al. A survey of newborn screening for cystic fibrosis in Europe. J Cyst Fibros. 2007;6:57-65.

6 Comeau AM, White TB, Campbell PW 3rd et al. Guidelines for implementation of cystic fibrosis newborn screening programs: Cystic Fibrosis Foundation workshop report. Pediatrics. 2007;119:e495-e518.

7 Castellani C, Southern KW, Brownlee K et al. European best practice guidelines for cystic fibrosis neonatal screening. J Cyst Fibros. 2010;8:153-73.

8 Brice P, Jarrett J, Mugford M. Genetic screening for cystic fibrosis: an overview of the science and the economics. J Cyst Fibros. 2007; 6:255-61.

9 Rosenstein BJ, Cutting GR, for the Cystic Fibrosis Foundation Consensus Panel. The diagnosis of cystic fibrosis: A consensus statement. J Pediatrics. 1998;132:589-95.

10 Farrell PM, Rosenstein BJ, White TB et al. Guidelines for diagnosis of cystic fibrosis in newborns through older adults: Cystic Fibrosis Foundation Consensus Report. J Pediatr. 2008; 153:S4-S14. 